\title{
Differences in Impact Factor Across Fields and Over Time
}

\author{
Benjamin M. Althouse, ${ }^{1}$ Jevin D. West, ${ }^{1}$ Theodore Bergstrom, ${ }^{2}$ and Carl T. Bergstrom ${ }^{1}$ \\ ${ }^{1}$ Department of Biology, University of Washington, Seattle, WA 98105 \\ ${ }^{2}$ Department of Economics, University of California Santa Barbara
}

\begin{abstract}
The bibliometric measure impact factor is a leading indicator of journal influence, and impact factors are routinely used in making decisions ranging from selecting journal subscriptions to allocating research funding to deciding tenure cases. Yet journal impact factors have increased gradually over time, and moreover impact factors vary widely across academic disciplines. Here we quantify inflation over time and differences across fields in impact factor scores and determine the sources of these differences. We find that the average number of citations in reference lists has increased gradually, and this is the predominant factor responsible for the inflation of impact factor scores over time. Field-specific variation in the fraction of citations to literature indexed by Thomson Scientific's Journal Citation Reports is the single greatest contributor to differences among the impact factors of journals in different fields. The growth rate of the scientific literature as a whole, and cross-field differences in net size and growth rate of individual fields, have had very little influence on impact factor inflation or on cross-field differences in impact factor.
\end{abstract}

\section{INTRODUCTION}

When Eugene Garfield published his 1972 paper in Science describing the role of impact factor in bibliometric studies, he provided a table of the highest-impact journals in science based on 1969 data. At that time, only 7 journals had impact factors of 10 or higher, and Science itself had an impact factor of 3.0 (1). Thirty five years later, in 2006, 109 journals have impact factors of 10 or higher, and Science registers an impact factor of 30.0 (2). Over the period from 1994, to 2005, the average impact factor of all journals indexed by Journal Citations Reports increased by about 2.6 percent per year.

Average impact factors differ not only over time, but across fields. For example, in 2006 the highest impact factor in the field of economics is 4.7 , held by the review journal Journal of Economic Literature. The top impact factor in molecular and cell biology is 47.4, held by $A n$ nual Reviews of Immunology. The average impact factors in these fields differ sixfold: the average impact factor in economics it is 0.8 whereas the average in molecular and cell biology is 4.8 .

This paper explores the sources of the increase in impact factor over the past 15 years, and the reasons for impact differences across fields. Citation and article counts were obtained from the CD-ROM version of the Thomson Journal Citation Reports (JCR) Science and Social Science editions, for the years 1994-2005.

\section{CHANGES IN IMPACT FACTOR OVER TIME}

A journal's impact factor is a measure of the number of times that articles published in a census period cite articles published during an earlier target window. The impact factor as reported by Thomson Scientific has a one year census period and uses the two previous years for the target window. Stated more formally, let $n_{t}^{i}$ be the number of times in year $t$ that the year $t-1$ and $t-2$ volumes of journal $i$ are cited. Let $A_{t}^{i}$ be the number of articles that appear in journal $i$ in year $t$. The impact factor $\mathrm{IF}_{t}^{i}$ of journal $i$ in year $t$ is

$$
\mathrm{IF}_{t}^{i}=\frac{n_{t}^{i}}{A_{t-1}^{i}+A_{t-2}^{i}}
$$

\section{A. Impact factors of individual journals}

The JCR database includes 4,300 journals that were indexed continually from 1994 to 2005. For these journals, Figure 1a plots 1994 impact factor scores against 2005 scores. Points above the diagonal represent journals with impact factor that have risen, and points below represent journals with impact factors that have fallen. About 80 $\%$ of the journals have increased in impact factor over the eleven years.

Figure $1 \mathrm{~b}$ shows the rank-order distribution of impact factors for years 1994 (lighter blue) through 2005 (darker blue). Impact factors scores increase annually, predominantly through the midrange of the distribution. From these figures, it is apparent that impact factors have increased steadily for most journals, independently of their initial impact factors.

\section{B. Weighted average impact factor}

To measure average rate of change, it is appropriate to assign larger weights to journals that publish more articles. The most convenient formulation assigns weights proportional to the number of articles that a journal published during the target years. Let $A_{t}^{i}$ be the number of articles published by journal $i$ in year $t$ and let $A_{t}$ be the sum of the articles published over the set $S_{t}$ of all journals indexed in year $t$.

We define the weight for journal $i$ in year $t$ as

$$
w_{t}^{i}=\frac{A_{t-1}^{i}+A_{t-2}^{i}}{A_{t-1}+A_{t-2}} .
$$


Comparison of IF in 1994 with IF in 2005 (Showing Density)

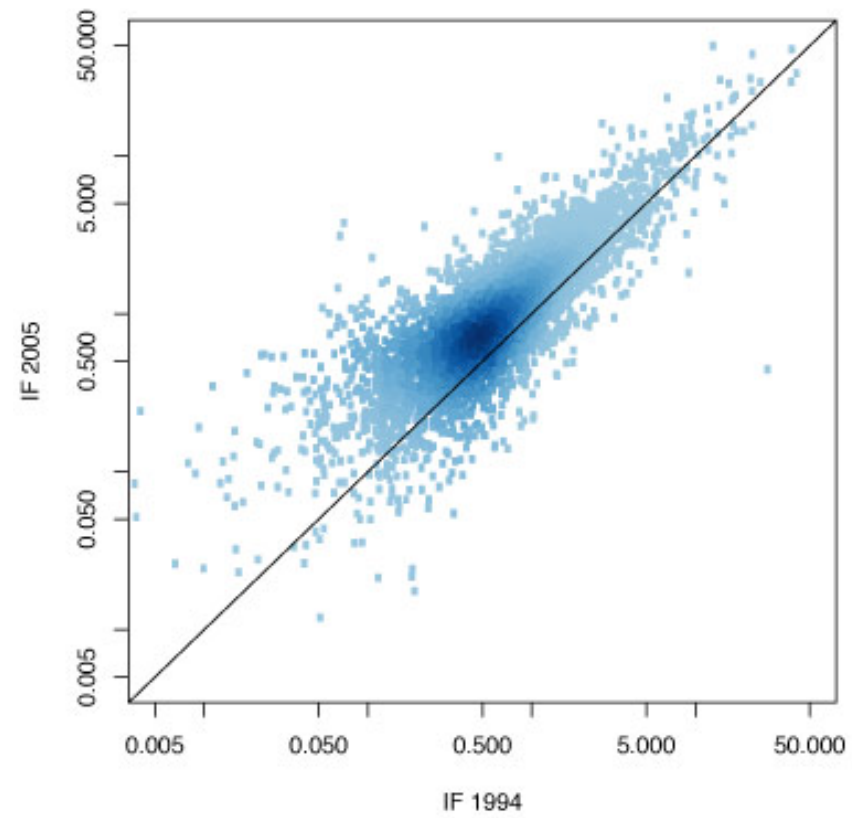

Rank Lists of IF (94-05)

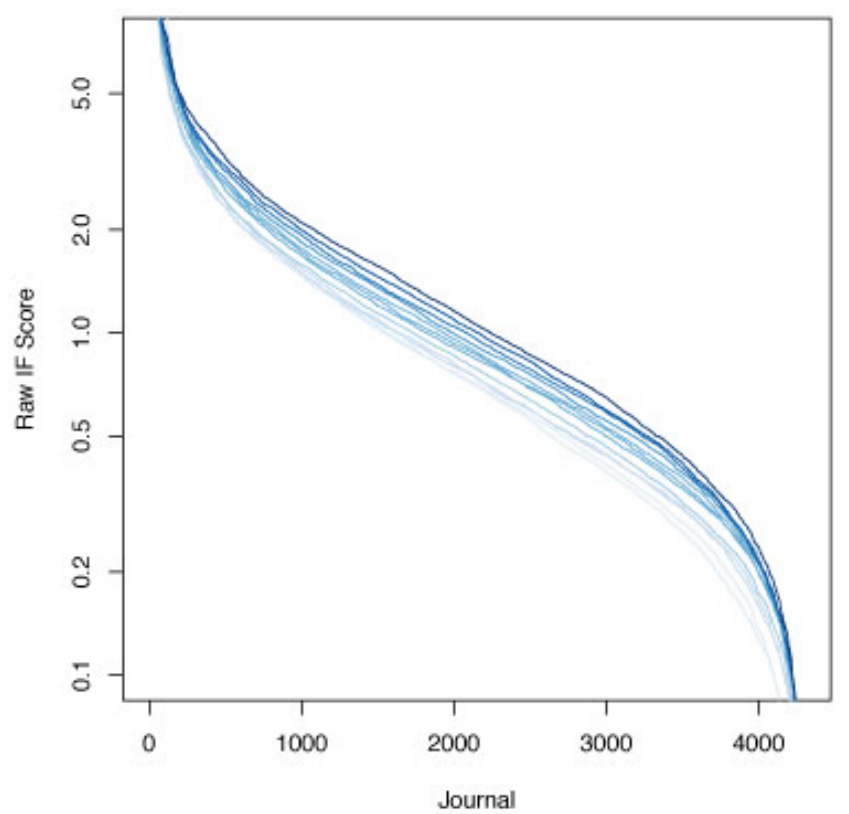

FIG. 1 Changes in impact factor from 1994 to 2005. Panel (a) is a log-log plot of 1994 impact factor against 2005 impact factor for the 4,300 journals that were listed continually from 1994 to 2005 in the JCR. Shading indicates density, with darker tones representing higher density. Panel (b) plots the rank-order distribution of impact factors from 1994 to 2005 . The progression of darkening shade indicates years, with the lightest shade representing 1994 and the darkest 2005.

Notice that $\sum_{i \in S_{t}} w_{t}^{i}=1$. Define the weighted average impact factor as

$$
\overline{\mathrm{IF}}_{t}=\sum_{i \in S_{t}} w_{t}^{i} \mathrm{IF}_{t}^{i}
$$

The weighted average impact factor for all journals listed in the JCR increased by an average rate of $2.6 \%$ percent per year from 1994 to 2005. For the journals that appeared in the index throughout the entire period from 1994 through 2005, the average annual increase was $1.6 \%$.

\section{Decomposing changes in average impact factor}

It might seem appealing to simply attribute the growth of impact factor to the growth of the scientific enterprise and in particular to the growth in the number of articles indexed by the JCR. The raw numbers lend a superficial plausibility to this view. From 1994 to 2005, the number of articles in JCR-indexed journals increased by $28 \%$ and the weighted impact factor increased by $29 \%$. But with a moment's reflection, we see that the connection is not immediate. For any given article, an increase in the number of related articles is a source of additional chances to be cited, but it is also a source of additional competition for the attention of potential readers and citations ${ }^{1}$. We will show that a constant rate of growth of the number of indexed articles will not result in increasing impact factors unless the number of citations per article also increases.

We have found a useful way to decompose the average impact factor in any period into the product of four factors. These are

1. The number of articles listed in the JCR, as measured by the ratio of number of articles published in the census period to the number of articles published in the target window.

2. The average number of citations in the reference list of each published article.

3. The fraction of all citations from articles written in the census period that cite articles published within the target window of the two prior years.

4. The fraction of cited articles published within the target window that appear in journals indexed by the JCR.

\footnotetext{
1 This point was observed by Garfield (3) who noted that there was no a priori reason to expect journals serving large scientific communities to have higher impact factors than those serving small ones.
} 
We construct this decomposition as follows. Let $c_{t}$ be the average number of papers cited by the journals in our dataset (i.e., the JCR-indexed journals) in year $t$. Let $p_{t}$ be the fraction of citations in our dataset in year $t$ that go to papers published in years $t-1$ and $t-2$. Let $v_{t}$ be the fraction of those citations appearing in our dataset in year $t$ and referencing items published in years $t-1$ and $t-2$, that go to journals that are listed in the JCR (as opposed to working papers, conference proceedings, books, journals not listed in the JCR, etc.).

Recalling our notation from Section II,

$$
\sum_{i \in S_{t}} n_{t}^{i}=A_{t} c_{t} p_{t} v_{t}
$$

and

$$
\begin{aligned}
\overline{\mathrm{IF}}_{t} & =\sum_{i \in S_{t}} w_{t}^{i} \mathrm{IF}_{t}^{i} \\
& =\sum_{i \in S_{t}} \frac{A_{t-1}^{i}+A_{t-2}^{i}}{A_{t-1}+A_{t-2}} \cdot \frac{n_{t}^{i}}{A_{t-1}^{i}+A_{t-2}^{i}} \\
& =\frac{\sum_{i \in S_{t}} n_{t}^{i}}{A_{t-1}+A_{t-2}} \\
& =\frac{A_{t} c_{t} p_{t} v_{t}}{A_{t-1}+A_{t-2}} .
\end{aligned}
$$

If we define $\alpha_{t}=A_{t} /\left(A_{t-1}+A_{t-2}\right)$, the weighted average impact factor at time $t$ can be written as the product

$$
\overline{\mathrm{IF}}_{t}=\alpha_{t} c_{t} p_{t} v_{t}
$$

The growth rate of a variable is approximated by the change in the logarithm of that variable. The multiplicative form of equation (6) makes it easy to decompose the growth rate of the average impact factor into the sum of growth rates of the variables $\alpha, c, p$, and $v$. It follows from equation (6) that

$$
\rho_{t}(\overline{\mathrm{IF}})=\rho_{t}(\alpha)+\rho_{t}(c)+\rho_{t}(p)+\rho_{t}(v),
$$

where for any variable $x$, we define $\rho_{t}(x)=\ln x_{t}-\ln x_{t-1}$. From the JCR data we are able to determine $\alpha_{t}, c_{t}, p_{t}$ and $v_{t}$, and hence $\rho_{t}(\alpha), \rho_{t}(c), \rho_{t}(p)$ and $\rho_{t}(v)$. Our methods for doing so are described in Appendix $\mathrm{A}$. The results are reported in Tables $\mathrm{I}$ and $\mathrm{II}$.

Somehow we have to account for an average increase in weighted impact factor of $2.6 \%$ per year over the period 1994-2005. Which of the four components is chiefly responsible? Table II lists the $\rho$ values for each component in each year; these $\rho$ values approximate the fractional increase due to each component in each year, and as such, provide the answer.

The increase in the number of articles published over the period 1994-2005 cannot explain the increase in impact factor over the same period. The values of $\rho_{t}(\alpha)$ are small in most years and this component contributes an overall average growth of -0.001 , i.e., impact factors would decline at an average of roughly 0.1 percent per

\begin{tabular}{c|c|c|c|c|c|c} 
Year $(t)$ & \# of articles & $\alpha_{t}$ & $c_{t}$ & $p_{t}$ & $v_{t}$ & $\overline{\mathrm{IF}}$ \\
\hline 1994 & 689,876 & 0.544 & 22.121 & 0.176 & 0.835 & 1.764 \\
1995 & 709,504 & 0.533 & 22.810 & 0.175 & 0.839 & 1.786 \\
1996 & 734,565 & 0.530 & 24.390 & 0.171 & 0.835 & 1.846 \\
1997 & 739,890 & 0.517 & 25.040 & 0.167 & 0.833 & 1.796 \\
1998 & 753,919 & 0.513 & 27.936 & 0.163 & 0.788 & 1.846 \\
1999 & 767,825 & 0.516 & 28.527 & 0.163 & 0.812 & 1.948 \\
2000 & 785,583 & 0.518 & 28.913 & 0.162 & 0.820 & 1.988 \\
2001 & 788,323 & 0.510 & 29.835 & 0.161 & 0.839 & 2.055 \\
2002 & 808,241 & 0.514 & 30.542 & 0.159 & 0.849 & 2.119 \\
2003 & 847,705 & 0.535 & 30.666 & 0.157 & 0.857 & 2.206 \\
2004 & 885,043 & 0.537 & 31.593 & 0.159 & 0.843 & 2.266
\end{tabular}

TABLE I Summary of time behavior of $\alpha_{t}, c_{t}, p_{t}$ and $v_{t}$ for the years 1994 to 2004 .

\begin{tabular}{c|c|c|c|c|c} 
Year $(t)$ & $\rho_{t}(\alpha)$ & $\rho_{t}(c)$ & $\rho_{t}(p)$ & $\rho_{t}(v)$ & $\rho_{t}(\overline{\mathrm{IF}})$ \\
\hline 1995 & -0.019 & 0.031 & -0.004 & 0.005 & 0.012 \\
1996 & -0.007 & 0.067 & -0.022 & -0.005 & 0.033 \\
1997 & -0.025 & 0.026 & -0.027 & -0.001 & -0.027 \\
1998 & -0.007 & 0.109 & -0.019 & -0.056 & 0.027 \\
1999 & 0.005 & 0.021 & -0.002 & 0.030 & 0.054 \\
2000 & 0.004 & 0.013 & -0.007 & 0.010 & 0.020 \\
2001 & -0.015 & 0.031 & -0.006 & 0.023 & 0.033 \\
2002 & 0.008 & 0.023 & -0.013 & 0.012 & 0.031 \\
2003 & 0.040 & 0.004 & -0.012 & 0.009 & 0.040 \\
2004 & 0.004 & 0.03 & 0.009 & -0.016 & 0.027 \\
\hline Mean & -0.001 & 0.036 & -0.010 & 0.001 & 0.025
\end{tabular}

TABLE II Summary of time behavior of $\rho_{t}(\alpha), \quad \rho_{t}(c), \quad \rho_{t}(p), \quad \rho_{t}(v)$ and $\rho_{t}(\overline{\mathrm{IF}})$ for the years 1995 to 2004 . The $\rho$ values approximate the fractional annual increase in each component $\alpha, c, p$, and $v$, and $\overline{\mathrm{IF}}$. The final row shows the average annual increase of each component over the period 1995-2004.

year if this were the only factor operating. The basic intuition underlying this result is as follows: first, note that larger fields do not have higher impact factors by shear virtue of their size. While more articles are published in larger fields and thus more citations are given out, those citations are shared among a larger pool of papers. Second, note that when a field grows at a constant rate, there will be more citation sources published year $t$ than citation targets published in year $t-1$, but this ratio of citation sources to citation targets will remain constant over time, and thus this difference will not inflate impact factors either.

We can show this formally. Suppose that the number of articles published grows at a constant rate $\gamma$ and that $c, p$, and $v$ remain constant. Then $A_{t}=(1+\gamma)^{t}$ and hence

$$
\begin{aligned}
\alpha_{t} & =\frac{(1-\gamma)^{t}}{(1-\gamma)^{t-1}+(1-\gamma)^{t-2}} \\
& =\frac{(1+\gamma)^{2}}{2+\gamma}
\end{aligned}
$$

Since $\alpha_{t}$ is constant, $\rho_{t}(\alpha)=0$ for all $t$.

Thus a constant rate of growth, $\gamma$, in the number of articles indexed annually leads to a constant impact fac- 
tor (no inflation). However, higher rates of growth will yield higher constant impact factors because the derivative of equation (8) with respect to $\gamma$ is positive. By contrast, accelerating growth in the number of articles published (increasing $\gamma$ over time) generates impact factor inflation and decelerating growth generates impact factor deflation. Changes in the other model parameters influence impact factor in more straightforward fashion. Likewise, increasing the average number of outgoing citations per article generates a corresponding increase in impact factor. Increasing the fraction of citations into the measurement window (the fraction of citations to JCRindexed literature in years $t-1$ and $t-2$ ) generates a corresponding increase in impact factor.

We cannot explain the increase in weighted impact factor by means of the change in the fraction of articles citing papers published within the recent two years. The $\rho_{t}(p)$ values are almost all negative, and in fact this component reduces the impact factor by an average of 1 percent per year over the period 1994-2005. In other words, impact factors would drop considerably if this were the only factor operating. Nor can we explain the increase in impact factor by changes in the fraction of citations to indexed articles. The average $\rho_{t}(v)$ is only 0.001 , i.e., the increase in the fraction of citations that reference indexed articles contributes only about 0.1 percent per year to the increase in impact factor.

This leaves as an explanation the change in the number of times the average article cites a reference source. Table I reveals a monotone increase in the average number of reference items cited $\left(c_{t}\right)$, and in Table 2 we see that this contributes large positive $\rho_{t}(c)$ values in each year, such that the average increase is approximately 3.6 percent, which adequately explains the $2.6 \%$ increase in weighted impact factor despite the net decline in due to the other components. In short, as citation practices change over time, the average number of citations per article is increasing, and the results is an inflation in impact factor over time.

Our analysis indicates that the single greatest contributor to impact factor inflation over the period 1994-2005 has been an increase in the average number of references per paper. One can imagine a number of potential causes for this increase. These include:

1. As the size of a field increases, the number of published papers that are relevant to any given manuscript might be expected to increase. Thus we might expect reference lists to grow longer as fields get bigger.

2. Internet search engines, on-line citation databases, and electronic access to the literature itself have considerably reduced the time-cost to authors of finding and obtaining relevant articles. This may have resulted in a concomitant increase in the number of cited items.
3. As researchers become increasingly aware of the value of citations to their own work, referees may demand that authors add numerous citations to their work, and authors may preemptively cite any number of potential editors and referees in their manuscript.

Preliminary regression analysis provided no evidence that increasing numbers of citable articles lead to increases in the length of reference lists. While it would interesting to seek out data that would allow us to distinguish among the other sources for the change in the average number of references per paper, we do not do so here.

\section{Natural Selection?}

During the period 1994-2005, the JCR added 4,202 new journals that were not previously listed and removed 2,415 journals that were listed in 1994. What effect, if any, did this process of journal substitution have on average impact factors? If the average impact factors of entering journals exceeded the average impact factor of exiting journals by a sizable margin, this could pull up the entire distribution. We could view this effect as a form of natural selection: the most fit - those with the highest impact factor scores - would enter or stay in the data set, while the least fit - those with the lowest scores - would drop out of the data set.

At first glance this seems to be plausible explanation. The journals that enter the JCR over the period 1995-2004 have significantly higher impact factor scores than those that exit over the same period (two sample Kolmogorov-Smirnov test, $\mathrm{D}=0.074, p=5.6 \mathrm{e}-7)$. However, even the entering journals had average impact factors well below the average for the full JCR. Because nearly twice as many journals entered as exited, the net effect of flux into and out of the JCR was actually to decrease the average impact factor of the full set of JCR listed journals.

We see this as follows. For a given year $t$, if we multiply the numbers of articles in years $t-1$ and $t-2$ by the overall weighted impact factor score for that year we can calculate the expected number of citations the set of entering or exiting journals would have to accrue in order to leave the average impact factor of the full set unchanged. The difference between the expected and the actual number of citations brought in by the entering journals can be considered a "citation cost" of adding new journals (whether positive or negative), and similarly the difference between the actual and the expected number of citations by journals exiting can be considered a "citation gain" of removing these journals from the data set. We can calculate then, the total effect of the flux of journals in and out of the data set by summing these quantities. For the years 1995-2004, an average cost of 18,200 citations per year was incurred due to turnover in 
the journals listed. Thus natural selection has not contributed to impact factor inflation.

While the journals that entered the JCR did not on average contribute to impact factor inflation by virtue of entering, they did contribute in the sense that subsequent to entering, their impact factors grew more strongly than did the average for the JCR as a whole. The average annual growth rate for those journals entering in years 1995-2004 is 6\%, more than twice the rate of the overall data set (see also (4)). Thomson is clearly selecting journals which are rising stars for inclusion in the JCR.

\section{DIFFERENCES IN IMPACT FACTOR ACROSS FIELDS}

Impact factors are well known to vary widely across disciplines (5; 6). Sources of this variation include differences in citation practices (7), differences in the lag time between publication and subsequent citation (what we call $p$ ) (7, 8), and differences in the proportions of citations directed to JCR-indexed literature (what we call $v$ ) (9; 10). Here we explore the source of these differences in detail. To delineate disciplinary boundaries, we use the field categories developed by Rosvall and Bergstrom (11). These categories use citation patterns to partition the sciences and social sciences into 88 non-overlapping fields.

Table III lists the 2004 weighted impact factors for the 50 largest fields. Indeed we see wide variation. For example, the field of Mathematics has a weighted impact factor of $\overline{\mathrm{IF}}=0.56$ whereas Molecular and Cell Biology has a weighted impact factor of 4.76 - an eight-fold difference. There are several possible sources of this difference, including but not limited to differences in growth rates, differences in the time course of citations, and differences in the fraction of citations that go to non-indexed literature. By extending the model developed in the previous section to partition the weighted impact factor into four separate contributing components, we can quantify the influence of each upon the cross-field differences.

To begin the analysis we recall Eq. (7):

$$
\rho_{t}(\overline{\mathrm{IF}})=\rho_{t}(\alpha)+\rho_{t}(c)+\rho_{t}(p)+\rho_{t}(v) .
$$

If journals received citations only from other journals in the same field, the following equation would hold exactly for each field $F$.

$$
\rho_{t}\left(\overline{\mathrm{IF}}_{F}\right)=\rho_{t}\left(\alpha_{F}\right)+\rho_{t}\left(c_{F}\right)+\rho_{t}\left(p_{F}\right)+\rho_{t}\left(v_{F}\right)
$$

In practice, not all citations come from within the same field, so the equation above is only approximate though it will be a very good approximation if most crossdisciplinary citations go between fields with similar $\alpha_{F}$, $c_{F}, p_{F}$, and $v_{F}$ values.

This will let us examine the influence on $\overline{\mathrm{IF}}$ of each component, $\alpha, c, p$, and $v$, in each field $F$ separately. How important is each component? A univariate linear regression of $\rho_{t}(\alpha), \rho_{t}(c), \rho_{t}(p)$, and $\rho_{t}(v)$ with $\rho_{t}(\overline{\mathrm{IF}})$ yields the following coefficients of determination $\left(r^{2}\right.$ values, indicating the proportion of total variability explained by each term):

$$
\begin{aligned}
r_{\alpha}^{2} & =0.045 \\
r_{c}^{2} & =0.172 \\
r_{p}^{2} & =0.083 \\
r_{v}^{2} & =0.456
\end{aligned}
$$

These coefficients of determination tell us a number of things. Firstly, the low value of $r_{\alpha}^{2}$ indicates that $\alpha_{t}$, the total number of articles in year $t$ over the total numbers of articles in years $t-1$ and $t-2$, explains very little of the variance across fields weighted impact factor. In contrast, the high value of $r_{v}^{2}$ indicates that the fraction of citations that go into ISI-listed material, $v_{F}$, explains the greatest fraction of variation of any of the four components.

If we progress to a multiple regression among pairs of variables, we find:

$$
\begin{aligned}
r_{\alpha, c}^{2} & =0.235 \\
r_{\alpha, p}^{2} & =0.118 \\
r_{\alpha, v}^{2} & =0.457 \\
r_{c, p}^{2} & =0.401 \\
r_{c, v}^{2} & =0.585 \\
r_{p, v}^{2} & =0.577
\end{aligned}
$$

This further demonstrates the minimal explanatory power of $\alpha: r_{\alpha, v}^{2}$ is approximately equal to $r_{v}^{2}$, and similarly for $r_{\alpha, c}^{2}$ and $r_{\alpha, p}^{2}$. It also confirms the considerable predictive power of $v$-any regression containing $v$ has a relatively high $r^{2}$, and shows that $c$ and $p$ are also predictively useful in concert with $v$. Multiple regressions with three and four variables yield:

$$
\begin{aligned}
r_{\alpha, c, p}^{2} & =0.451 \\
r_{\alpha, c, v}^{2} & =0.591 \\
r_{\alpha, p, v}^{2} & =0.577 \\
r_{c, p, v}^{2} & =0.854 \\
r_{\alpha, c, p, v}^{2} & =0.855
\end{aligned}
$$

The $r^{2}$ with all four variables is 0.855 ; the model is unable to perfectly predict the weigted impact factor because our assumption that all citations received come from the same field is not strictly true. Noice also that $r_{\alpha, c, p, v}^{2} \cong r_{c, p, v}^{2}$, further indicating that $\alpha$ has little, if any, predictive power.

The method of Hierarchical Partitioning (12) provides a more formal method to estimate the relative contributions or "importance" of the various independent vari- 
ables in explaining the total explained variance in a multivariate regression. The statistic $I$ estimates the contribution of each independent variable. Using the hierarchical partitioning hier . part package by Chris Walsh in the statistical analysis program R, we find the following $I$ values for the year 2004 data.

\begin{tabular}{c|c} 
Predictor & $\mathrm{I}(\%)$ \\
\hline$\alpha$ & 2.858 \\
$c$ & 26.624 \\
$p$ & 20.178 \\
$v$ & 50.340
\end{tabular}

These results indicate that the predictor $v$ (the fraction of citations to JCR-indexed literature) accounts for $50 \%$ of the explained variance $\overline{\mathrm{IF}}$. The predictor $c$ (number of outgoing citations per article) accounts for an additional $27 \%$. Those fields which cite heavily within the ISI data set, such as Molecular Biology or Medicine, buoy their own scores. Those fields which do not cite heavily within the ISI data set such as Computer Science or Mathematics have correspondingly lower scores.

Figure 2 summarizes the differences in weighted average impact factor across fields (panel d) and the factors responsible for these differences (panels $\mathrm{a}-\mathrm{c}$ ).

\section{A. Inflation differences across fields}

As we have shown in previous sections, weighted impact factor is increasing every year and is different for each field. Naturally, the next several questions to be asked are Is inflation ubiquitous across fields? Do some fields inflate more than others? Which fields inflate the most? Differences in inflation rates between fields will be important when evaluating citation data within a specific field over time. Knowing that, for instance, psychiatry is inflating twice as fast as neuroscience, would help one compare journals across these fields over time.

The results of the analysis are reported in Table III. Fields vary substantially in their rates of impact factor inflation. Further analysis shows that inflation rate is not correlated to size of field $\left(r^{2}=0.001\right)$, nor weighted impact factor scores of that field $\left(r^{2}=0.018\right)$.

\section{Summary}

Impact factors vary across fields and over time. By decomposing average impact factors into four contributing components - field growth, average number of cited items per paper, fraction of citations to papers published within two years, and fraction of citations to JCR-listed items - we are able to determine the sources of this variation. We find that an increasing number of citations in the reference lists of published papers is the greatest contributor to impact factor inflation over time. Differences in the fraction of citations to JCR-indexed literature is the greatest contributor to differences across fields, though cross-field differences in impact factor are also influenced by differences in the number of citations per paper and differences in the fraction of references that were published within two years. By contrast, the growth rate of the scientific literature and cross-field differences in net size and growth rate have very little influence on impact factor inflation or on cross-field differences in impact factor.

\section{Competing interests}

The authors are the developers of Eigenfactor (http://www.eigenfactor.org), a method for ranking journal influence using citation network data.

\section{Acknowledgments}

The authors would like to thank Martin Rosvall for generating the maps used in Figure 2, and also Alan Wilson for drawing out attention to impact factor inflation. This work was supported in part by a Mary Gates Research Scholarship and a Howard Hughes Medical Institute Integrative Research Internship to B.M.A.

\section{APPENDIX A: Deriving $\alpha_{t}, c_{t}, p_{t}$ and $v_{t}$ from the JCR data}

All citation data sets come from the JCR data sets for the years 1994 through 2005. The JCR does not list article counts for year $t$ in data set for year $t$; the year $t+1$ and year $t+2$ data sets typically do not agree exactly on the number of articles that were published in year $t$. Therefore, in order to compute the the year $t$ article count, $\mathrm{A}_{t}$, we average the article count listed for year $t$ in the $t+1$ data set and year $t$ in the $t+2$ data set. We then calculate $\alpha_{t}=\mathrm{A}_{t} /\left(\mathrm{A}_{t-1}+\mathrm{A}_{t-2}\right)$ using the total article counts for years $\mathrm{A}_{t-1}$ and $\mathrm{A}_{t-2}$ as given in the data set for year $t$.

We calculate $c_{t}$ by dividing the total outgoing citations for all journals in year $t$ by the total articles for year $t$ :

$$
c_{t}=\frac{\text { total out-citations in year } t}{\mathrm{~A}_{t}} .
$$

We calculate $p_{t}$ by dividing the total outgoing citations for all journals to material published in the previous two years $(t-1$ and $t-2)$ by the total outgoing citations for all journals in year $t$ :

$$
p_{t}=\frac{2 \text {-year total out-citations from year } t}{\text { total out-citations in year } t} .
$$

The calculation of $v_{t}$ is slightly more complicated than the other calculations; Figure 3 provides a schematic representation. To calculate the percentage of citations into the JCR for the entire dataset we divide the total incoming citations for the previous two years (figure 3 top 

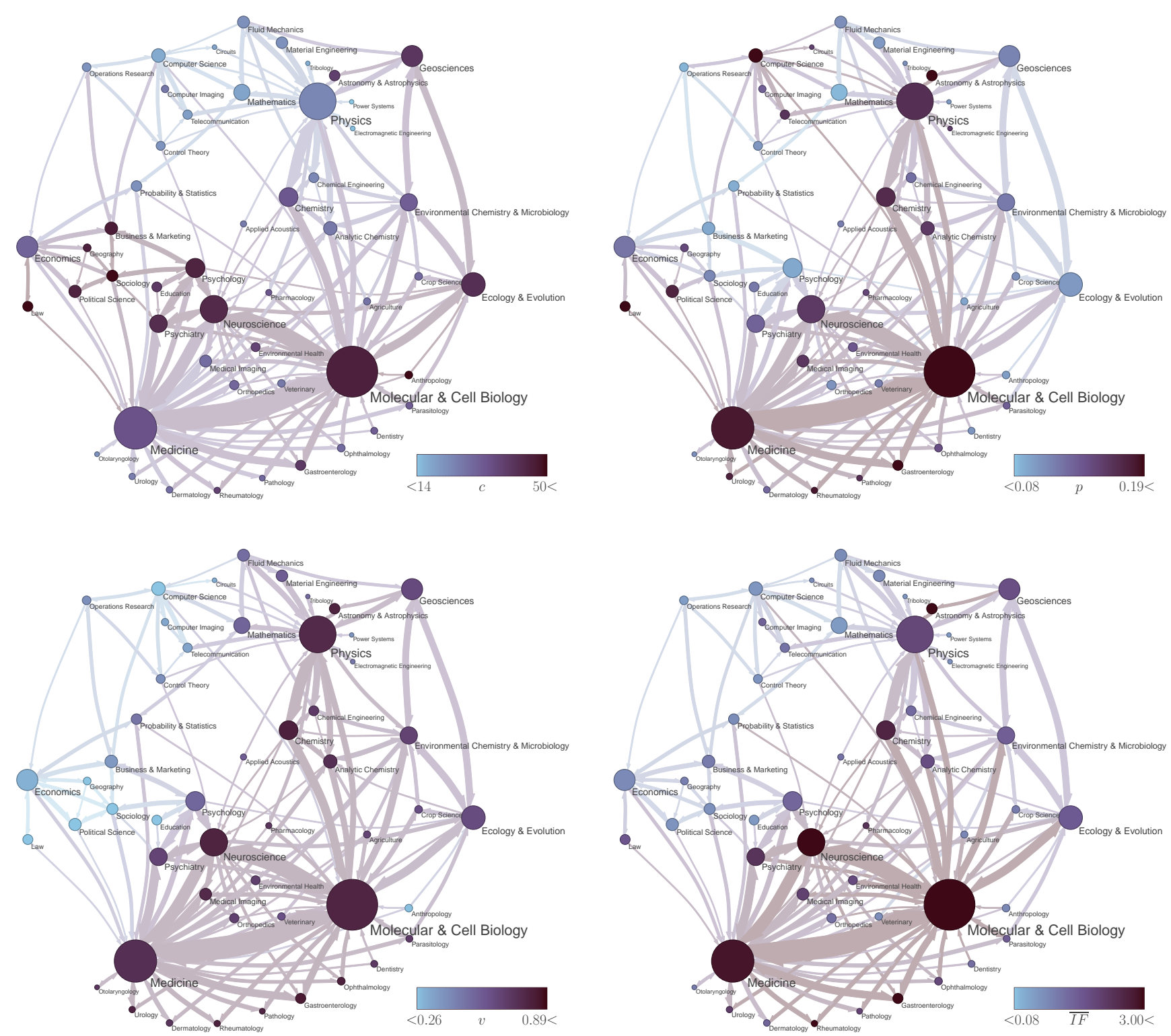

FIG. 2 Differences in citation pattern across fields. Fields are categorized and mapped as in Rosvall and Bergstrom (2008). Panel a: average number of items cited per paper. Panel b: Fraction of citations to papers published in the two previous calendar years. Panel c: Fraction of citations to papers published in JCR-listed journals. Panel d: Weighted impact factor, $\overline{\mathrm{IF}}$.

panel, arrow A) by the total outgoing citations over that period (arrows A $+\mathrm{C}$ ):

$v_{(t, \text { Entire Dataset })}=\frac{2 \text {-year total in-citations from year } t}{2 \text {-year total out-citations from year } t}$.

This is done because the incoming citations for the entire dataset are the outgoing citations from the JCR to itself. However, this is not true for the specific field calculations. To calculate $v_{t, F}$ for any field $F$, we divide the 2-year outgoing citations from that field to itself (figure 3, bottom panel, arrow A) plus the 2-year outgoing citations from that field to the rest of the JCR (arrow
B) by the total 2-year outgoing citations from that field (arrows $\mathrm{A}+\mathrm{B}+\mathrm{C})$ :

$$
v_{(t, F)}=\frac{\left(\begin{array}{l}
2 \text {-year out-citations from } F \text { to } F+ \\
2 \text {-year out-citations into rest of JCR }
\end{array}\right)}{\text { 2-year total out-citations from year } t}
$$

\section{References}

[1] E. Garfield, Citation Analysis as a Tool in Journal Evaluation, Science 178:471-79, 1972. 


\begin{tabular}{|c|c|c|c|c|c|c|}
\hline Field (Size) & $\overline{\mathrm{IF}}$ & $\alpha$ & $c$ & $p$ & $v$ & $\begin{array}{l}\text { Growth } \\
\text { Rate }\end{array}$ \\
\hline Molecular and Cell Biology (511) & 4.763 & 0.515 & 45.810 & 0.205 & 0.803 & 0.006 \\
\hline Astronomy and Astrophysics (25) & 4.295 & 0.530 & 38.249 & 0.215 & 0.813 & 0.074 \\
\hline Gastroenterology (40) & 3.475 & 0.494 & 39.669 & 0.193 & 0.849 & 0.030 \\
\hline Rheumatology (20) & 3.348 & 0.519 & 37.818 & 0.184 & 0.826 & 0.079 \\
\hline Neuroscience (224) & 3.252 & 0.515 & 43.768 & 0.159 & 0.810 & 0.017 \\
\hline Medicine (766) & 2.896 & 0.515 & 33.920 & 0.183 & 0.760 & 0.036 \\
\hline Chemistry (145) & 2.610 & 0.539 & 33.103 & 0.170 & 0.821 & 0.026 \\
\hline Pharmacology (28) & 2.331 & 0.575 & 32.947 & 0.149 & 0.737 & 0.098 \\
\hline Psychiatry (178) & 2.294 & 0.522 & 43.025 & 0.131 & 0.670 & 0.039 \\
\hline Urology $(23)$ & 2.132 & 0.513 & 25.501 & 0.176 & 0.806 & 0.032 \\
\hline Medical Imaging (84) & 2.043 & 0.502 & 28.727 & 0.161 & 0.784 & 0.034 \\
\hline Pathology (28) & 1.991 & 0.516 & 29.523 & 0.166 & 0.803 & 0.020 \\
\hline Physics (503) & 1.912 & 0.543 & 23.963 & 0.167 & 0.783 & 0.018 \\
\hline Ophthalmology (36) & 1.905 & 0.536 & 29.105 & 0.144 & 0.823 & 0.029 \\
\hline Environmental Health (73) & 1.871 & 0.533 & 37.234 & 0.140 & 0.691 & 0.048 \\
\hline Analytic Chemistry (129) & 1.789 & 0.538 & 26.702 & 0.158 & 0.762 & 0.022 \\
\hline Geosciences $(224)$ & 1.768 & 0.526 & 40.529 & 0.113 & 0.647 & 0.021 \\
\hline Law $(71)$ & 1.657 & 0.485 & 76.826 & 0.199 & 0.231 & 0.010 \\
\hline Ecology and Evolution (349) & 1.555 & 0.523 & 42.172 & 0.100 & 0.640 & 0.051 \\
\hline Parasitology (38) & 1.527 & 0.505 & 32.076 & 0.134 & 0.711 & 0.036 \\
\hline $\begin{array}{l}\text { Environmental Chemistry } \\
\text { and Microbiology (181) }\end{array}$ & 1.505 & 0.518 & 31.648 & 0.117 & 0.679 & 0.039 \\
\hline Computer Imaging (31) & 1.446 & 0.514 & 26.470 & 0.133 & 0.332 & 0.067 \\
\hline Dermatology (38) & 1.427 & 0.480 & 28.442 & 0.128 & 0.734 & 0.050 \\
\hline Psychology (210) & 1.387 & 0.513 & 45.139 & 0.091 & 0.538 & 0.033 \\
\hline Chemical Engineering (75) & 1.290 & 0.587 & 23.660 & 0.124 & 0.711 & 0.041 \\
\hline Dentistry (43) & 1.284 & 0.529 & 32.046 & 0.102 & 0.717 & 0.029 \\
\hline Orthopedics (72) & 1.226 & 0.531 & 30.033 & 0.103 & 0.683 & 0.066 \\
\hline Telecommunication (37) & 1.192 & 0.550 & 19.518 & 0.163 & 0.334 & 0.054 \\
\hline Applied Acoustics (36) & 1.171 & 0.526 & 25.942 & 0.115 & 0.575 & 0.031 \\
\hline Crop Science $(61)$ & 1.040 & 0.523 & 29.467 & 0.104 & 0.631 & 0.025 \\
\hline Business and Marketing (101) & 1.035 & 0.538 & 46.865 & 0.091 & 0.376 & 0.032 \\
\hline Geography (56) & 0.986 & 0.526 & 46.055 & 0.148 & 0.254 & 0.029 \\
\hline Information Science (23) & 0.918 & 0.539 & 28.402 & 0.220 & 0.217 & 0.078 \\
\hline Agriculture (56) & 0.882 & 0.530 & 27.503 & 0.093 & 0.670 & 0.024 \\
\hline Anthropology (62) & 0.872 & 0.496 & 52.104 & 0.098 & 0.275 & 0.020 \\
\hline Material Engineering (107) & 0.826 & 0.537 & 22.038 & 0.100 & 0.578 & 0.063 \\
\hline Economics (159) & 0.823 & 0.511 & 30.423 & 0.121 & 0.299 & 0.021 \\
\hline Fluid Mechanics (107) & 0.804 & 0.520 & 22.096 & 0.107 & 0.516 & 0.041 \\
\hline Probability And Statistics (57) & 0.796 & 0.528 & 21.974 & 0.089 & 0.496 & 0.023 \\
\hline Veterinary $(77)$ & 0.767 & 0.480 & 26.512 & 0.115 & 0.620 & 0.041 \\
\hline Sociology $(96)$ & 0.715 & 0.510 & 50.840 & 0.110 & 0.189 & 0.001 \\
\hline Media and Communication (24) & 0.690 & 0.479 & 46.932 & 0.133 & 0.190 & 0.024 \\
\hline Control Theory (64) & 0.681 & 0.474 & 21.394 & 0.102 & 0.407 & 0.061 \\
\hline Political Science (99) & 0.680 & 0.500 & 45.014 & 0.176 & 0.131 & 0.012 \\
\hline Computer Science (124) & 0.631 & 0.717 & 17.215 & 0.193 & 0.266 & 0.034 \\
\hline Education (86) & 0.590 & 0.509 & 39.890 & 0.119 & 0.213 & 0.015 \\
\hline Mathematics (149) & 0.556 & 0.512 & 18.477 & 0.085 & 0.552 & 0.033 \\
\hline Operations Research (62) & 0.542 & 0.521 & 21.714 & 0.086 & 0.408 & 0.043 \\
\hline $\begin{array}{l}\text { History and Philosophy } \\
\text { Of Science }(32)\end{array}$ & 0.456 & 0.507 & 51.316 & 0.068 & 0.159 & -0.003 \\
\hline History (23) & 0.416 & 0.466 & 81.775 & 0.101 & 0.059 & -0.028 \\
\hline
\end{tabular}

TABLE III Table showing $\alpha, c, p, v$ and exponential growth rates for individual fields. All except growth rate were calculated using 2004 data.

[2] Journal Citation Reports (1994-2005), Thomson Scientific.

[3] E. Garfield, The History and Meaning of the Journal Impact Factor, JAMA 295:90-93, 2006.

[4] A. Wilson, Journal Impact Factors are Inflated, Bio-
Science, Vol. 57, No. 7, 2007

[5] P. Seglen, Why the impact factor of journals should not be used for evaluating research. British Medical Journal, 314:497, 1997.

[6] P. Vinkler, Bibliometric features of some scientific sub- 

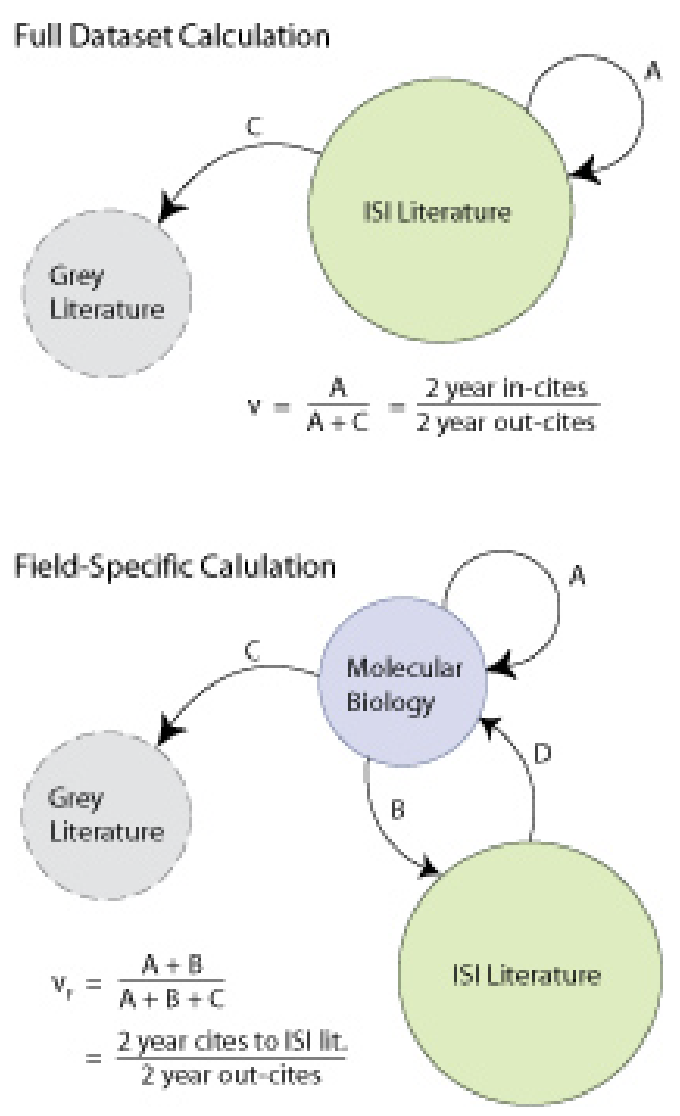

FIG. 3 Calculating $v_{t}$. Top panel gives the schematic for calculating $v_{t}$ for the entire dataset, and the bottom panel gives the schematic for specific fields. fields and the scientometric consequences therefrom, Scientometrics, Vol. 14, Nos. 5-6, 1988.

[7] H.F. Moed, W.J.M. Burger, J.G. Frankfort, A.F.J. Van Raan, The application of bibliometric indicators: important field and time-dependent factors to be considered, Scientometrics, Vol. 8, Nos. 3-4, 1985.

[8] J. Marton, Obsolescence or Immediacy? Evidence Supporting Price's Hypothesis, Scientometrics, Vol. 7, Nos. 3-6, 1985

[9] D. Hamilton, Research Papers: Who's Uncited Now? Science, 251:25, 1991

[10] J. Vanclay, Bias in the Journal Impact Factor, Scientometrics, in press, 2007.

[11] M. Rosvall and C. T. Bergstrom, Maps of random walks on complex networks reveal community structure, Proceedings of the National Academy of Sciences USA 105:1118-1123.

[12] A. Chevan and M. Sutherland, Hierarchical Partitioning, The American Statistician, Vol. 45, No. 2., pp. 90-96, May 1991. 